\title{
CFD computation of ship motions and added resistance for a high speed trimaran in regular head waves
}

\author{
Cheng-sheng Wu, De-cai Zhou, Lei Gao and Quan-ming Miao
}

China Ship Scientific Research Center, Wu-xi, Jiang-su, China

\begin{abstract}
Some research work on CFD computation of ship motions and added resistance in waves for a high speed trimaran is carried out in this paper. The governing equations, Reynolds Averaged Navier-Stokes and continuity equations are discretized by finite volume method. Volume of fluid method is adopted to deal with the nonlinear free surface. The incident waves are generated from the inflow boundary by prescribing a velocity profile resembling flexible flap wavemaker motions, and the outgoing waves are dissipated inside an artificial damping zone located at the rear part of the wave tank. In this paper, the computed results of ship motion and added resistance for a high speed trimaran are presented. The results of seakeeping experiment for the high speed trimaran carried out in CSSRC towing tank are also presented in this paper. Rather good agreements are shown between the computational and experimental results. The work in this paper provides a numerical tool for the study of seakeeping performance of high speed trimarans.
\end{abstract}

KEY WORDS: High speed trimaran; Ship motion; Added resistance in waves; CFD computation.

\section{INTRODUCTION}

The trimaran configuration may offer some advantages over conventional hull forms. It is widely accepted that both wave-making and form resistance decrease as a vessel becomes more slender. Because of the stability gained form the side hulls, the trimaran can adopt a more slender main hull that can greatly reduce residuary resistance. Having three separate hulls on a trimaran creates a higher total wetted surface area compared to a similar mono-hull. This higher wetted surface increases the frictional resistance therefore creating comparatively higher resistance at low speeds. At high speeds the wave-making and form resistance is relatively low due to the use of slender hulls. Wave-making resistance is also affected by the interference between the separate hull wakes. Optimum placement of the side hulls will result in a wake interference that reduces this resistance. The combination of a slender hull form and optimum placement of side hulls can result in a much lower resistance at high speeds when compare to mono-hull design.

The trimaran may also have some advantages in waves. The distances between the side hulls and the main hull provide a relatively long arm of force for roll, which can reduce the roll motion of the ship. The slender main hull and side hulls placed at the aft part of the ship create a

Corresponding author: Cheng-sheng $\mathrm{Wu}$

e-mail: cswu@163.com higher inertial moment of pitch, which can reduce the pitch motion. Some investigations proved that the performance of heave motion in waves is also fine. The better performance of ship motions may results in lower added resistance in waves. Other advantages of the trimaran include increase in deck space, increase in stability and passenger comfort and so on.

Because of the trimaran having so many advantages, a lot of research work has been done during the last decades, include the studies on hydrodynamics performance. Most of the research work on hydrodynamics focused on the optimization of placement of side hulls to get an optimum interference between hulls and lower resistance (Brizzolara et al., 2005, Sahoo and Lawrence, 2005, Yang et al., 2002). However, with the increase of high-speed trimaran operating in the world, the capability to evaluate the behavior of such vessels in waves becomes more demanding. In general, the model test (Brizzolara et al., 2003) and numerical prediction (Chou et al., 2008, Sato et al., 2007) are common approaches to investigate seakeeping characteristics.

Some research work on CFD computation of ship motions and added resistance in waves for a high speed trimaran is carried out in this paper by solving RANSE (Reynolds Averaged Navier-Stokes Equations). The governing equations are discretized by finite volume method. Volume of fluid method is adopted to deal with the nonlinear free surface. In this paper, the computed results of ship motion and added resistance for a high speed trimaran are presented. The results of seakeeping experiment for the high 
speed trimaran carried out in CSSRC (China Ship Scientific Research Center) towing tank are also presented in this paper. Rather good agreements are shown between the computational and experimental results.

\section{MATHEMATICAL MODEL}

\section{Governing Equations}

Numerical simulations in this paper are carried out based on numerical wave tank (Wu et al., 2008). The nonlinear free surface flow is treated as two-phase flow, i. e. water and air, free surface is the interface between air and water, and volume of fluid method is adopted to deal with it. Both water and air are assumed to be incompressible and isotropic Newtonian fluid. The governing equations for the NWT include continuity equation, volume fraction equation and momentum equation.

The continuity equation is,

$$
\nabla \cdot \vec{V}=0
$$

where $\vec{V}$ is the velocity vector.

Because of the fluid is assumed to be incompressible, the conservation equation of water (or air) in the two-phase flow of waves can be written in the form of volume fraction,

$\frac{\partial \alpha_{w}}{\partial t}+\vec{V} \cdot \nabla \alpha_{w}=0$

In every control volumes, the summation of volume fraction of water and air should be one,

$$
\alpha_{w}+\alpha_{a}=1
$$

in which $\alpha$ is volume fraction, the subscripts $a$ and $w$ denote air and water respectively.

The conservation equation of momentum for the twophase flow is,

$$
\frac{\partial}{\partial t}(\rho \vec{V})+\nabla \cdot(\rho \vec{V} \vec{V})=-\nabla p+\nabla \cdot\left[\mu\left(\nabla \vec{V}+\nabla \vec{V}^{T}\right)\right]+\rho \vec{g}
$$

where $\rho$ is the density, $\mu$ is the viscosity, $\vec{g}$ is the gravity acceleration, $p$ is pressure. The density of the two-phase flow $\rho$ is given by,

$\rho=\alpha_{w} \rho_{w}+\alpha_{a} \rho_{a}$

The viscosity and some other physical parameters are computed in the same manner.

RNG $k$ - $\varepsilon$ two-equation model is employed to model turbulence.

\section{Wave Generation and Absorption}

The incident waves are generated from the inflow boundary by prescribing a wavy velocity profile. According to the linear wave theory in water of infinite depth, the free surface elevation of regular wave should be,

$\eta=A \cos (k x-\omega t)$

And the velocity field of regular wave flow will be,

$\left\{\begin{array}{l}U=\omega A e^{k z} \cos (k x-\omega t) \\ V=0 \\ W=\omega A e^{k z} \sin (k x-\omega t)\end{array}\right.$

where $A$ is the wave amplitude, $k$ is the wave number, $\omega$ is the radian frequency.

The outgoing waves are dissipated inside an artificial damping zone located at the rear part of the wave tank. The artificial damping added to the momentum equation (4) can be expressed as,

$D m p=\left\{0,0, \gamma\left(\frac{x-x_{s}}{x_{e}-x_{s}}\right)^{2}\left(\frac{z_{b}-z}{z_{b}-z_{f s}}\right)\right\} \cdot \vec{V}$

in which $\gamma$ is the control parameter. The subscripts $s$ and $e$ denote the start and end points of the artificial damping zone in the $x$-direction, $b$ and $f s$ denote the bottom and free surface position of the wave tank in the $z$-direction respectively. In the damping zone, the mesh size is gradually increased in the horizontal direction to provide additional numerical damping.

\section{Ship Motion Computation}

The ship motion is analyzed in the global coordinate system. The governing equations of the six degrees of freedom (6DOF) of a rigid body motion can be expressed as,

$\frac{d}{d t}\left(m \vec{v}_{C}\right)=\vec{F}$

$\frac{d}{d t}\left(\boldsymbol{M}_{C} \cdot \vec{\omega}_{C}\right)=\vec{m}_{C}$

where the subscript $C$ denotes the center of mass of the ship. $m$ denotes the mass of the ship, $\vec{v}_{C}$ the velocity vector, $\boldsymbol{M}_{\boldsymbol{C}}$ the tensor of the moments of inertia, $\vec{\omega}_{C}$ the angular veloctiy vector, $\vec{F}$ the resulting force vector and $\vec{m}_{C}$ the resultant moment vector acting on the ship and can be computed by,

$\vec{F}=\int_{S}([\tau]-p[I]) \cdot \vec{n} d S-m \vec{g}$ 
$\vec{m}_{C}=\int_{S}\left(\vec{r}-\vec{r}_{C}\right) \times([\tau]-p[I]) \cdot \vec{n} d S$

where $[\tau]$ is the shear stress tensor acting on the surface of the ship hulls, $\vec{n}$ the outward nomal vector of hulls' surface, $\vec{r}_{C}$ denotes the location vector of the center of gravity of the ship and $\vec{r}$ the location vetor of the point on the hulls' surface.

Once the forces and moments acting on the ship are known, the velocities and positions of the ship can be obtained by solving and integrating Eqs. (9) and (10).

\section{Couple of Flow and Ship Motion}

The forces and moments acting on the surface of ship, which strongly influence the ship motion, are obtained from the flow as described above. However, the flow is influenced by ship motion as well and both problems have to be considered simultaneously. The coupled procedure of flow and body motion consists of the following steps:

1) Provide the initial values for the dependent variables at the time $t_{0}$.

2) Advance the time by $\Delta t$.

3) Assemble and solve by an iterative solver the linearized algebraic equations for the velocity components in turn, employing the currently available other dependent variables.

4) Assemble and solve the algebraic equations for the pressure correction and correct mass fluxes, velocity components, and pressure.

5) Assemble and solve the algebraic equations for volume fraction $c$ and use the calculated values to update wave surfaces and the properties of the effective fluid.

6) Assemble and solve the algebraic equations for turbulent kinetic energy $k$ and its dissipation rate $\varepsilon$ and obtain turbulent diffusion coefficients.

7) Integrate the pressure and shear forces over ship surfaces, compute field forces.

8) Solve and integrate the resultant equations of motion for the ship.

9) Adapt the grid to the new position of the body.

10) Return to Step 3 and repeat until the sum of absolute residuals for all equations has fallen by prescribed number of orders of magnitude.

11) Return to Step 2 and repeat until the prescribed number of time steps is completed.

The couple of flow and ship motions in wave is summarized briefly as the Fig. 1 .

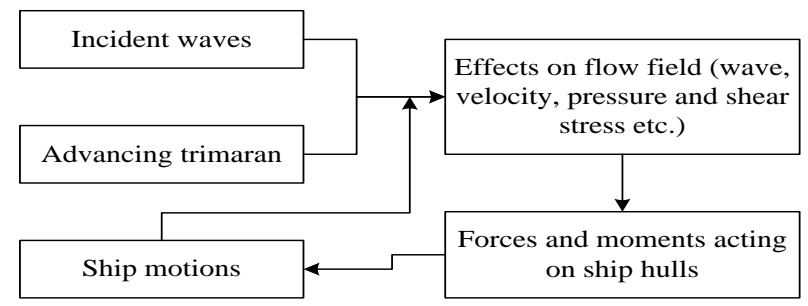

Fig. 1 Couple of ship motions and flow.

\section{NUMERICAL SIMULATION}

\section{Computational Domain and Grid}

The origin of the coordinate for the computational domain locates on the intersection of free-surface of calm water, symmetric plane and vertical-transverse mid-plane of the main hull. The computational domain extents are: $1.8 L \sim 4.0 L$ in $x$-direction include damping zones with length of about $2.5 L,-1.2 L \sim 1.2 L$ in $y$-direction and $-1.2 L \sim 0.4 L$ in $z$ direction, where $L$ denotes the ship length. The outline of the computational domain is shown in Fig. 2.

The computational domain is discretized by multi-block structural grid. There are at least 30 cells per wave length in $x$-direction, 10 20cells within the region of wave height and 60 cells in the extent of the width of the computational domain. The computational grid is also shown in Fig. 2.

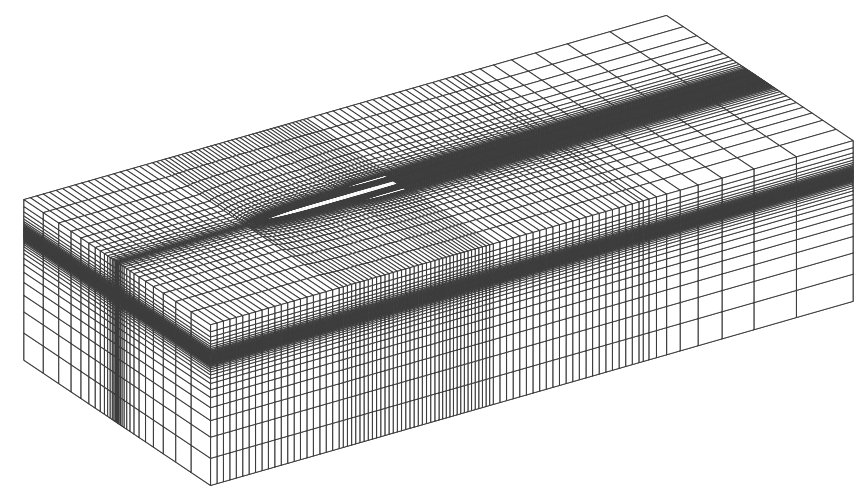

Fig. 2 Computational Domain and Grid.

\section{Boundary Conditions}

The boundary of the computational domain is composed of inlet boundary, outlet boundary, wall boundary (hulls' surface of the trimaran), and outer boundary (include bottom, proof and side of the domain). The detailed boundary conditions are listed below.

- On the inlet boundary, a velocity profile resembling flexible flap wavemaker motions and volume fraction are prescribed.

- On the outlet boundary, the free surface here is assumed to be calm after the waves pass through the artificial damping zone, the hydrostatic pressure is set.

- On the surface of ship hulls, the standard wall function is introduced.

- On the outer boundary, zero stress is specified.

\section{Numerical Scheme and Solver}

The governing equations are discretized by finite volume method (FVM), second-order upwind difference scheme was adopted for the convection term and the centric difference scheme for the dissipation term. Multigrid acceleration algorithm and SIMPLE (Semi-Implicit Method for Pressure Linked Equations) are used to solve the difference equation system. 


\section{TEST CASE}

A trimaran model advancing in regular head waves of amplitude $(A)$ about $0.03 m$ and the wave length $(\lambda)$ varies from $0.5 L$ to $3.0 L$ with different speeds corresponding to Froude number $(\mathrm{Fr}) 0.52$ and 0.70 respectively, are simulated in this paper. The configuration of the trimaran is shown in Fig. 3. The inertial radius of pitch is set as $0.25 \mathrm{~L}$.
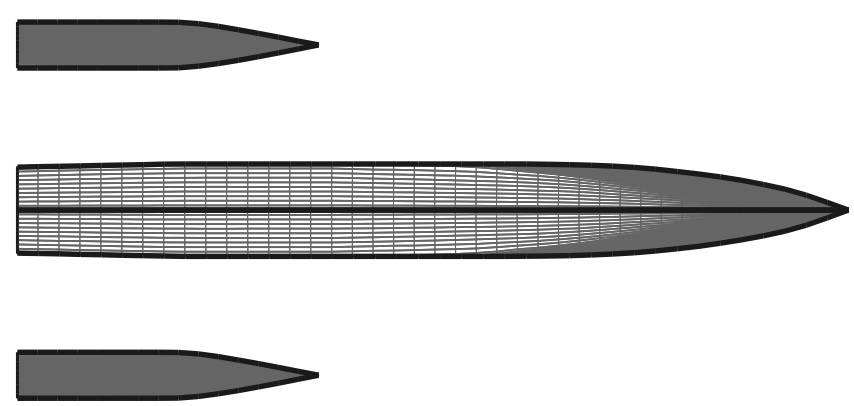

Fig. 3 Configuration of the Trimaran

\section{COMPUTATIONAL RESULTS}

The model is free to heave and pitch in the simulations,

$$
\left\{\begin{array}{l}
z=z_{a} \cdot \cos \left(\omega_{e} t+\varepsilon_{z}\right) \\
\theta=\theta_{a} \cdot \cos \left(\omega_{e} t+\varepsilon_{\theta}\right)
\end{array}\right.
$$

in which $z_{a}$ and $\theta_{a}$ are amplitudes of heave and pitch motions respectively, $\omega_{e}$ is the encounter radian frequency.

The nondimensional amplitudes of heave and pitch motions are,

$$
\left\{\begin{array}{l}
z_{a}^{\prime \prime}=z_{a} / A \\
\theta_{a}^{\prime \prime}=\theta_{a} /(k A)
\end{array}\right.
$$

Let $\bar{R}_{W V}$ and $R_{S W}$ denote the averaged resistances in waves and calm water respectively. Then the added resistance in waves for the trimaran model will be,

$$
R_{a w}=\bar{R}_{W V}-R_{S W}
$$

The nondimensional added resistance can be expressed as,

$$
R_{a w}^{\prime \prime}=\frac{R_{a w}}{\rho_{w} g A^{2} B^{2} / L}
$$

in which B is the breadth of the main hull of the trimaran.

Fig. 4 shows the typical time series of heave and pitch motions for the numerical simulations. It can be observed that the time histories reach a steady state after about 5 cycles and the profiles almost maintain uniform shapes after that.
The experiments of ship motions and added resistance in regular head waves for the trimaran model have been carried out in CSSRC's towing tank with dimensions of $474 \mathrm{~m}$ in length, $14 \mathrm{~m}$ in width and $7 \mathrm{~m}$ in depth. One flap-type wave generator system is installed at the end of the tank. Regular and irregular waves can be generated. The effective length can be used for seakeeping test is about $200 \mathrm{~m}$.
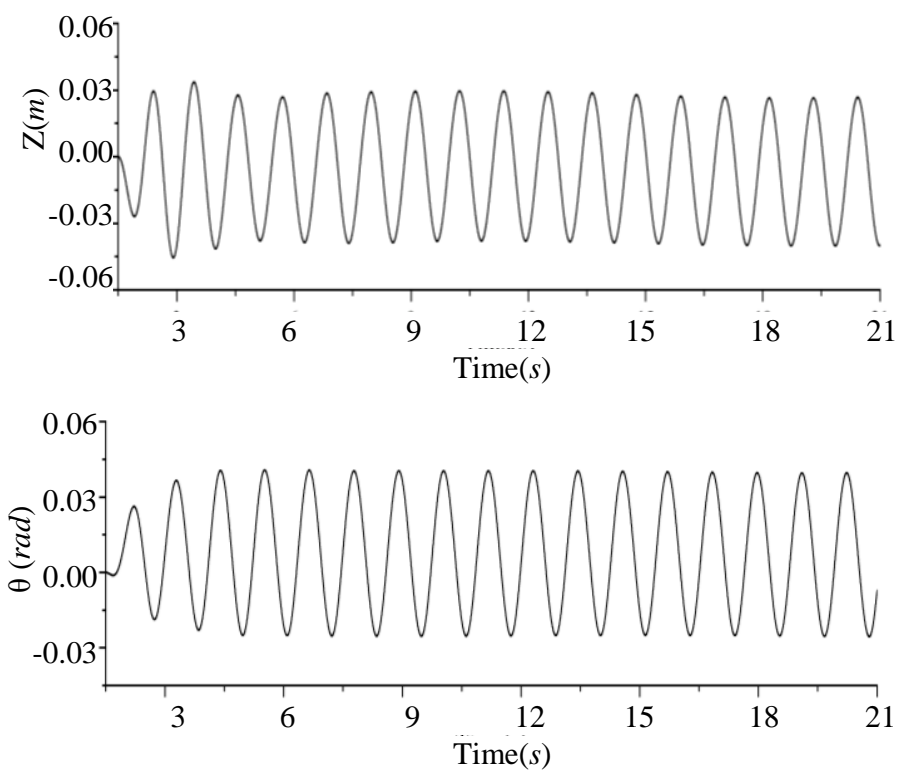

Fig. 4 Time Series of Heave and Pitch Motions.

The simulated heave transfer functions of the trimaran model with different speeds are shown in Fig. 5. The experimental results are also plotted in the figure. From the figures we can see that the heave motions of numerical simulations agree rather well with those of experiment in general. The differences between numerical and experimental results are acceptable, although the heave motion of the trimaran are over-predicted at a lot of wave length conditions.

Fig. 6 gives the pitch transfer functions. The experimental results are also plotted in the figure. The figures indicate that the pitch motions of numerical simulations agree quite well with those of experiment. The differences between numerical and experimental results are small and acceptable, although the pitch motions of the trimaran are over-predicted or underpredicted at a few wave length conditions.

The computed added resistance transfer functions of the trimaran model with different speeds are shown in Fig. 7. The experimental results are also plotted in the figure. The figures indicate that the CFD computations predict the similar tendency with the experimental data for added resistance of trimaran in head waves. But the numerical simulations underpredicted the added resistance near the peaks of the transfer function curves.

The wave patterns of the trimaran advancing in head waves for $\lambda / L=1.5$ at $F r=0.52$ and 0.70 are shown in Fig. 8 . From the figure we can see the interaction between the waves excited by the advancing ship, ship motions, ship diffraction, the interference between the hulls and the incident regular waves. 

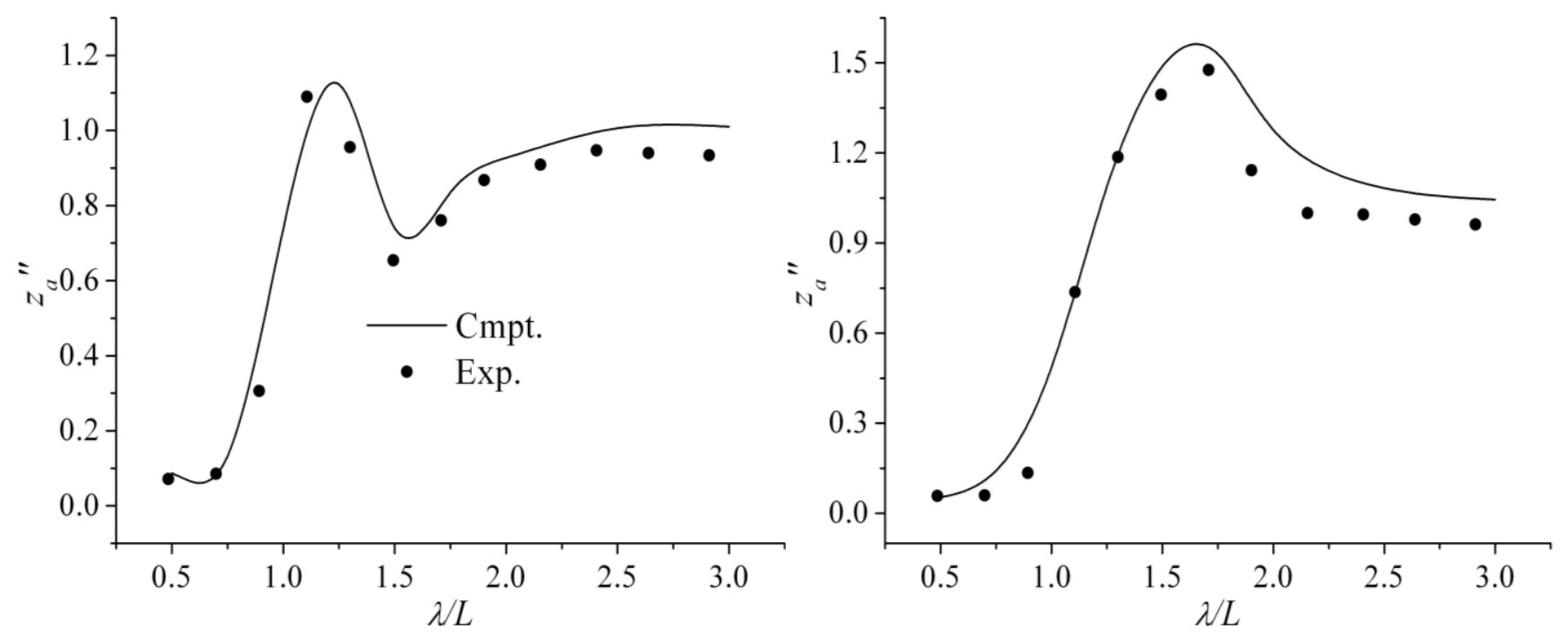

Fig. 5 Heave Transfer Function of the Trimaran.
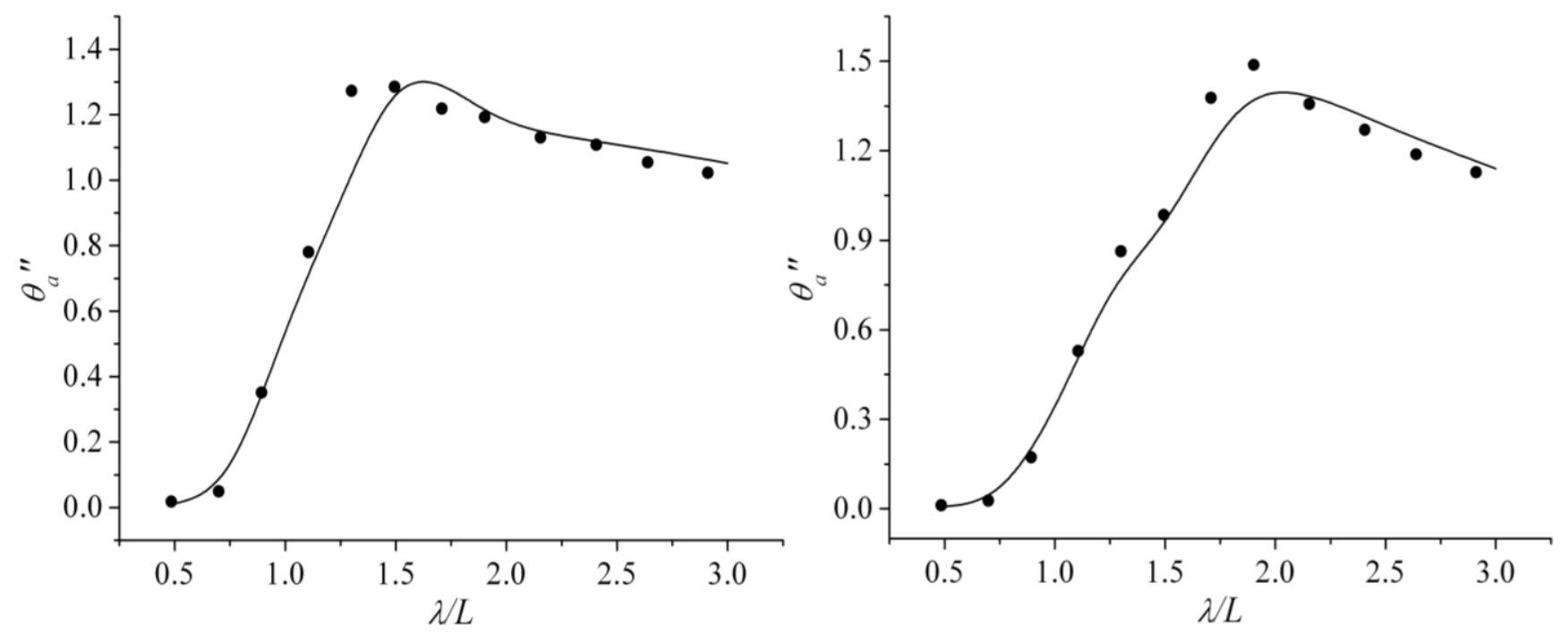

Fig. 6 Pitch Transfer Function of the Trimaran.
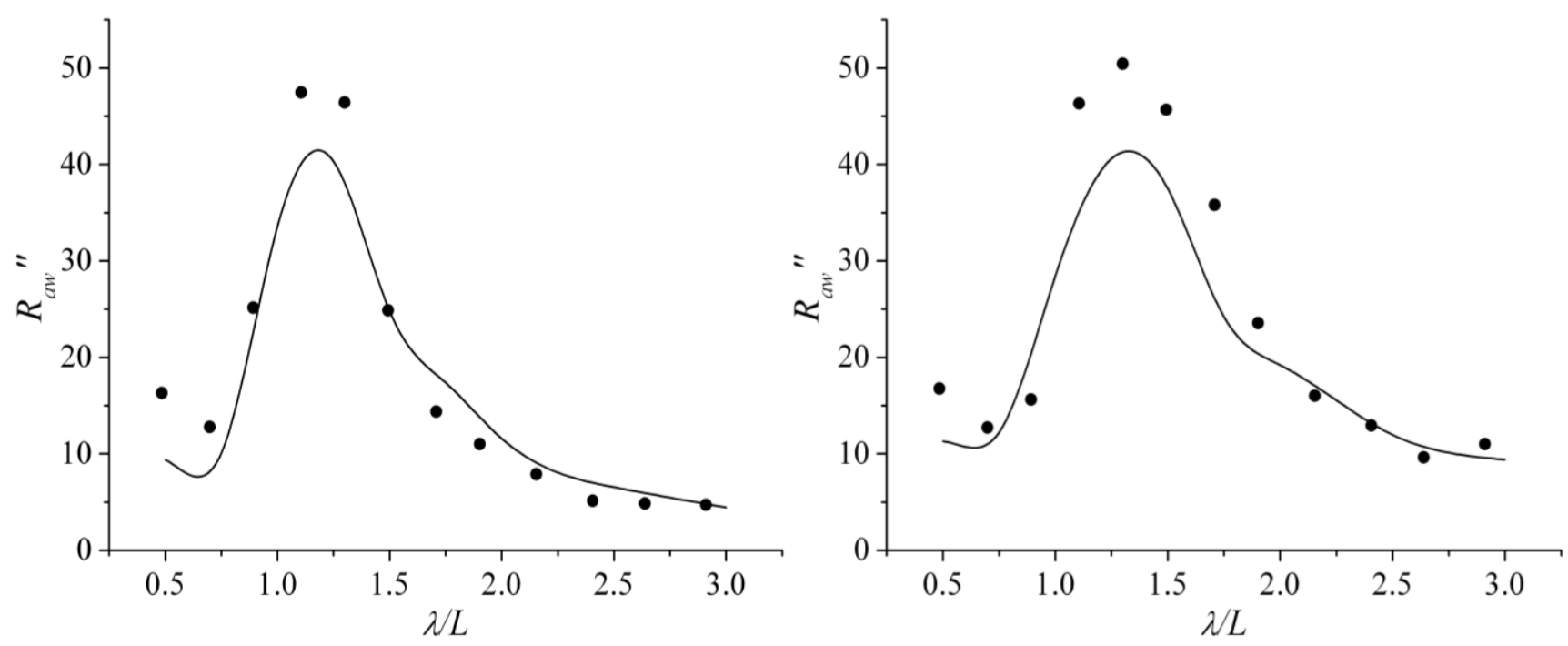

Fig. 7 Added Resistance Transfer Function of the Trimaran. 

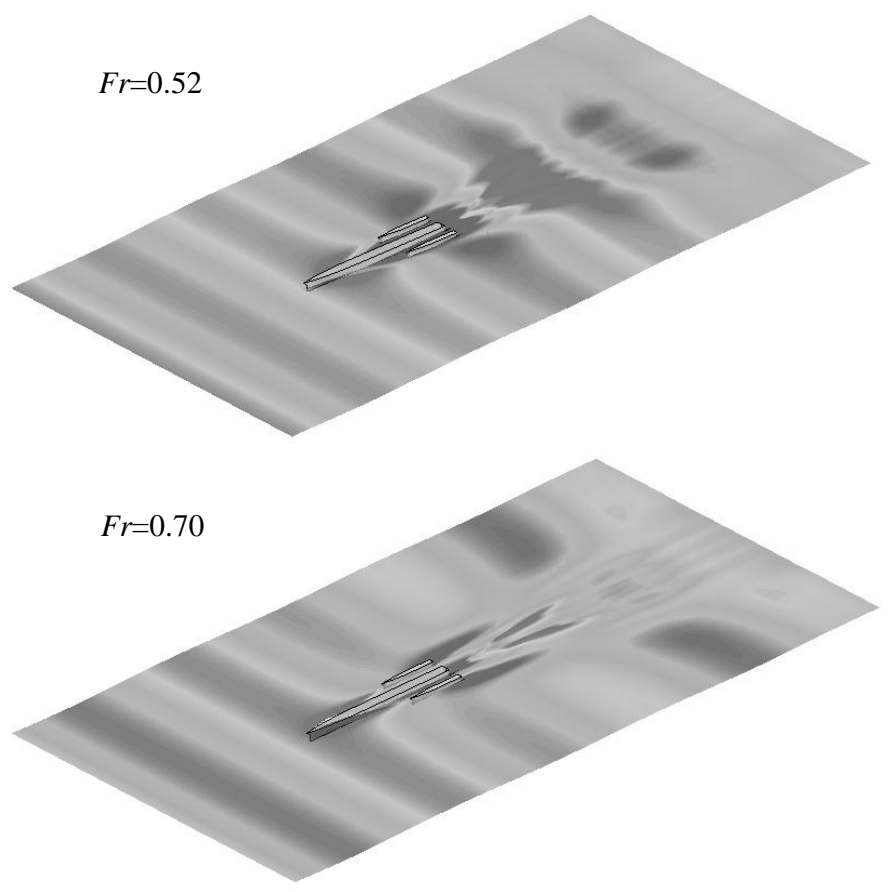

Fig. 8 Wave Patterns for $\lambda / L=1.5$.

In general, wave induced motions and added resistance for trimaran advancing in regular head waves can be predicted quite well by using the numerical method presented in this paper. Most of the differences between numerical and experimental results are acceptable.

\section{CONCLUDING REMARKS}

Some research work on CFD computation of ship motions and added resistance in waves for a high speed trimaran is carried out in this paper by solving RANS equations. Numerical simulations of wave induced motions and computation of added resistance for a trimaran advancing in regular head waves are conducted by present numerical method. The CFD predictions of ship motions include heave and pitch are compared with the experimental data from CSSRC and show quite good agreements.
CFD computations predict the similar tendency with the experimental data for added resistance. On the whole, most of the differences between numerical and experimental results are acceptable.

The work in this paper provides a numerical tool for the study of seakeeping performance of high speed trimarans. Further work should be done to improve the present numerical method and to upgrade the accuracy of the predictions for wave induced motions and added resistances, especially for added resistances near the peaks of the transfer function curves.

\section{REFERENCES}

Brizzolara, S. Capasso, M. and Francescutto, A., 2003. Effect of hulls form variations on the hydrodynamic performances in waves of a trimaran ship. Proceedings of the $7^{\text {th }}$ International conference on Fast Sea Transportation, pp. 7-14. Athens. Greece.

Brizzolara, S. Capasso, M. and Francescutto, A., 2005. Effect of hulls form variations on hydrodynamic performances of a trimaran ship for fast transportation. Proceedings of the $7^{\text {th }}$ International conference on Fast Sea Transportation, Saint Petersburg, Russia.

Chou, S.K. Wu, H.T. Wu, C.H. Hwang, J.L., 2008. Investigation on seakeeping performance of the highspeed trimaran. Proceedings of the $8^{\text {th }}$ National Congress on Hydrodynamics, Ji-nan, China.

Sahoo, P.K. and Lawrence, J., 2005. The waves generated by a trimaran. Proceedings of the $8^{\text {th }}$ International Conference on the Fast Sea Transportatioin, Saint Petersburg, Russia.

Sato, Y. Uzawa, K. and Miyata, H., 2007. Validation of motion prediction method for trimaran vessels. Proceedings of the $9^{\text {th }}$ International Conference on Numerical Ship Hydrodyanmics, Ann Arbor, MI, USA.

Wu, C. Zhu, D. and Gu, M., 2008 Development of a viscous numerical wave tank and simulation of wave induced motions for a ship in regular head waves. Proceedings of the $8^{\text {th }}$ International Conference on Hydrodynamics (ICHD2008), Nantes, France.

Yang, C. Soto, O. Löhner, R. Noblesse, F., 2002 Hydrodynamic optimization of a trimaran. Ship Technology Research, 49(2), 70-92. 